\title{
Auswertung der Umfrage der ÖOG-Kommission für Kataraktchirurgie und intraokulare Implantate für 2020
}

\author{
Guenal Kahraman $(D)$ Kataraktkommission der ÖOG
}

Eingegangen: 11. November 2021 / Angenommen: 16. November 2021 / Online publiziert: 21. Dezember 2021

(c) The Author(s), under exclusive licence to Springer-Verlag GmbH Austria, ein Teil von Springer Nature 2021

\begin{abstract}
Zusammenfassung
Hintergrund Auch im Jahr 2020 wurde die jährliche Umfrage durch die ÖOG-Kommission für Kataraktchirurgie und intraokulare Implantate unter den österreichischen Augenabteilungen durchgeführt.

Material und Methoden Die Umfrage erfolgte mit Hilfe anonymer schriftlicher Fragebögen, welche alternativ auch per Online-Formular ausgefüllt werden konnten. Die Teilnahme an der Umfrage ist freiwillig. Dies ist bei der Interpretation der Ergebnisse zu berücksichtigen. Es erfolgte eine statistische Auswertung der aktuellen Fragebögen, die mit den Ergebnissen der Vorjahre korreliert wurden.

Ergebnisse Die Angaben von 18 Operationszentren mit insgesamt 149 Operateuren werden hier vorgestellt und mit den Ergebnissen der Vorjahre verglichen. Die Teilnehmerquote lag bei $72,4 \%$. Insgesamt wurden 52.059 Kataraktoperationen erfasst. Es zeigt sich, dass die tagesklinischen Eingriffe im Vergleich zum Vorjahr um 24,8\% gesunken sind. Dies ist vermutlich auf die Restriktionen von elektiven Operationen im Rahmen der SARS-CoV-2-Pandemie zurückzuführen.
\end{abstract}

Schlüsselwörter Katarakt · Intraokularlinse · Kataraktoperation · Tagesklinische Operation

Die Mitglieder der Kataraktkommission der ÖOG werden am Beitragsende gelistet.

G. Kahraman $(\bowtie)$

Akademisches Lehrkrankenhaus der Barmherzigen Brüder Wien, Johannes von Gott Platz 1, 1020 Wien, Österreich

Medizinische Fakultät, Sigmund Freund Privatuniversität Wien, Freudplatz 3, 1020 Wien, Österreich

kahraman@gmx.at

\author{
Evaluation of the survey of the Austrian \\ Ophthalmic Society, Commission for Cataract \\ Surgery and Intraocular Implants for 2020
}

\section{Summary}

Background Every year the Austrian Commission for Cataract Surgery and Intraocular Implants, conducts a national survey of all eye surgery departments in Austria.

Material and methods The questionnaires were sent to departments of ophthalmology in Austria and were anonymously returned either printed or via an online form. Before analyzing the results it is important to consider that participation was voluntary and compared to the data of previous years.

Results The responses of 18 departments of ophthalmology with a total of 149 are presented in this publication and combined with data from previous years.

The participation rate was $72.4 \%$ with a total of 52,059 cataract surgeries reported. Compared to the previous year this amounts to a decrease of $24.8 \%$; however, this is most likely caused by restrictions of elective surgery in association with the SARS-CoV-2 pandemic.

Keywords Cataract · Intraocular lens · Cataract statistic · Outpatient surgery

Die Kommission für Kataraktchirurgie und intraokulare Implantate führt seit 2006 eine österreichweite Umfrage über die Anzahl und Art der Kataraktoperationen durch. Diese wird jährlich sowohl in schriftlicher Form, als auch per Online-Formular an die Primarärzt/Innen aller augenchirurgischen Abteilungen in Österreich verschickt. 
Tab. 1 Ergebnis der Befragung

\begin{tabular}{|l|l|l|l|l|}
\hline Chirurgen & Operationen Zugangsort Inzisionsgröße & Endophthalmitisfälle \\
\hline 2 & 531 & Superior & 2,2 & 0 \\
\hline 5 & 820 & Superior & 2,5 & 0 \\
\hline 6 & 3245 & Superior & 2,4 & 0 \\
\hline 2 & 123 & On axis & 2,2 & 0 \\
\hline 10 & 1499 & Superior & 2,4 & 12 \\
\hline 14 & 3758 & Superior & 2,2 & 45 \\
\hline 8 & 4000 & Superior & 2,4 & 15 \\
\hline 11 & 2842 & Temporal & 2,2 & 60 \\
\hline 6 & 2465 & Superior & 2,4 & 14 \\
\hline 13 & 3601 & Superior & 2,5 & 41 \\
\hline 6 & 2400 & On axis & 2,4 & 20 \\
\hline 15 & 2903 & Superior & 2,4 & 21 \\
\hline 1 & 535 & Superior & 2,4 & 0 \\
\hline 5 & 3430 & Temporal & 2,5 & 28 \\
\hline 13 & 8650 & Temporal & 2,4 & 60 \\
\hline 9 & 3200 & On axis & 2,5 & 7 \\
\hline 11 & 1772 & Superior & 2,4 & 23 \\
\hline 12 & 6285 & Superior & 2,4 & 24 \\
\hline
\end{tabular}

Tab. 2 Die meisten Zentren bevorzugen eine 2,4-mm-Inzisionsgröße

\begin{tabular}{|l|l|}
\hline Inzisionsgröße & Absolute Häufigkeit \\
\hline 2,5 & 4 \\
\hline 2,4 & 10 \\
\hline 2,2 & 4 \\
\hline
\end{tabular}

Tab. 3 Bevorzugte Anästhesiemethode

\begin{tabular}{|l|l|l|}
\hline Tropfanästhesie/intrakameral & Absolute Zahlen & Prozent \\
\hline Peribulbär, parabulbär, retrobulbär & 45.510 & 88 \\
\hline Allgemeinanästhesie & 4485 & 9 \\
\hline
\end{tabular}

Folgende Parameter werden ermittelt:

- Anzahl der Kataraktoperationen,

- Anzahl der operierenden Augenärzte,

- Anteil der tageschirurgischen Operationen,

- Methode der Anästhesie,

- Art der implantierten Intraokularlinse,

- Anzahl an explantierten IOLs und Explantationsgrund,

- Antibiotikaprophylaxe,

- Dauer und Art der postoperativen Medikation.

Für das Jahr 2020 wurden 24 Fragebögen an alle augenchirurgischen Zentren verschickt, davon wurden $18(72,4 \%)$ retourniert, diese kamen hauptsächlich aus öffentlichen Spitälern, eine Einreichung erfolgte durch ein Sanatorium oder Privatspital. Eine Übersicht findet sich in Tab. 1. Insgesamt wurden im Jahr 202052.059 Kataraktoperationen von 149 Chirurg/ Innen durchgeführt; $45.586(84,576 \%)$ waren tagesklinische Eingriffe. Im Vergleich dazu fanden im Vorjahr 69.194 (2019) Kataraktoperationen statt. Somit zeigt

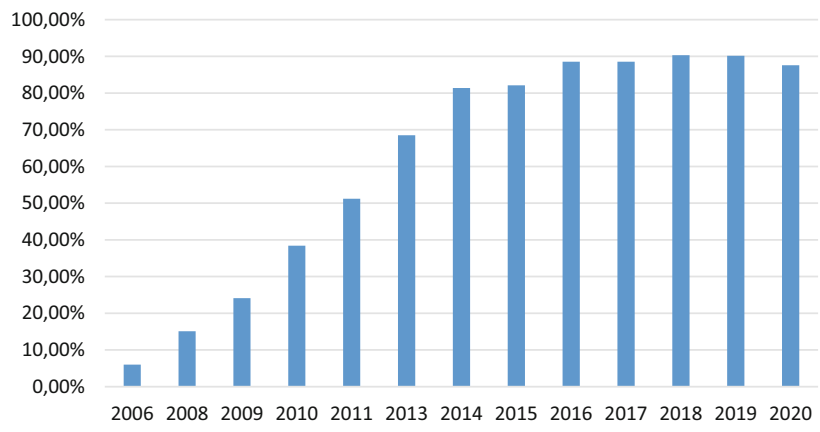

Abb. 1 Entwicklung der tagesklinischen Kataraktoperationen

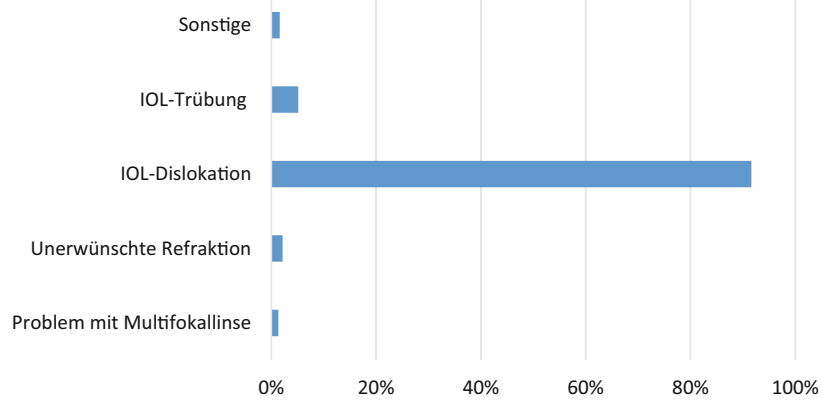

Abb. 2 IOL-Explantationsgründe

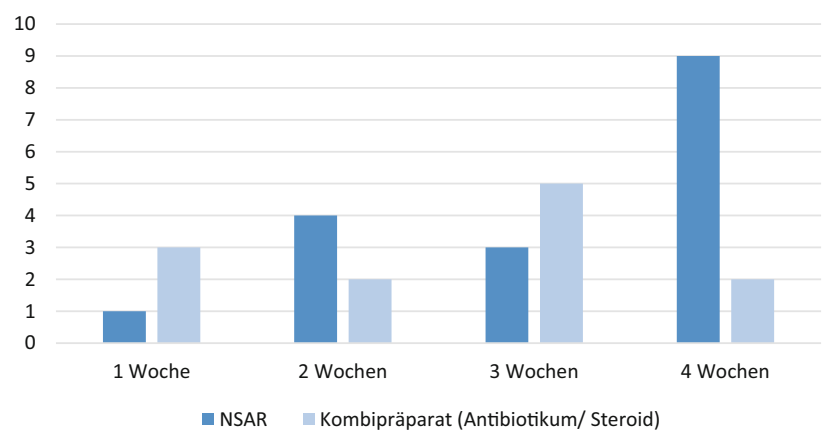

Abb. 3 Postoperative Therapiedauer

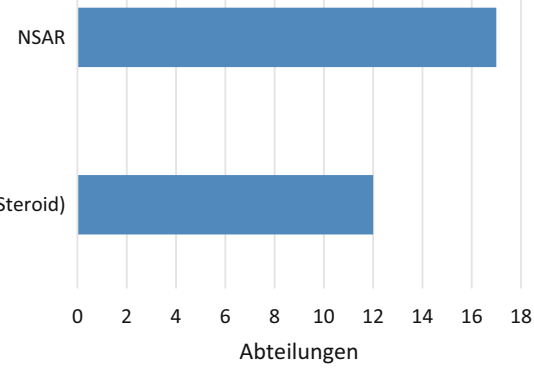

Abb. 4 Postoperative Therapie

sich eine deutliche Regredienz der Operationen. Diese ist jedoch vermutlich auf die SARS-CoV-2-Pandemie und die damit verbundene Reduktion elektiver Operationen zurückzuführen. Auch die Zahl der tagesklinischen Eingriffe ist in diesem Jahr etwas geringer (Abb. 1). 
Tab. 4 Bevorzugtes Linsenmaterial

\begin{tabular}{|c|c|c|c|c|c|c|c|c|}
\hline [Angaben in \%] & 2013 & 2014 & 2015 & 2016 & 2017 & 2018 & 2019 & 2020 \\
\hline Hydrophob Acryl & 75,4 & 80,8 & 88,5 & 88,6 & 88,61 & 90,7 & 89 & 62,4 \\
\hline Hydrophil Acryl & 12,6 & 10,4 & 7,5 & 7,49 & 6,3 & 7,49 & 9 & 37,2 \\
\hline Silikon & 11,5 & 8,5 & 3,8 & 3,8 & 3,8 & 1,3 & 2 & 0,1 \\
\hline PMMA & 0,2 & 0,1 & 0,2 & 0,02 & 0,015 & 0,01 & 0 & 0,3 \\
\hline $\begin{array}{l}\text { Andere/ohne Anga- } \\
\text { be }\end{array}$ & 0,3 & 0,2 & 0 & 0 & 0,11 & 0,5 & 0 & 0 \\
\hline
\end{tabular}

Im Jahr 2020 gaben 13 (72\%) Abteilungen „Clear cornea (,obligat unblutig')" als bevorzugten Zugangsschnitt an, 5 (28\%) Abteilungen bevorzugten „Limbal (,blutig')“; 12 (67\%) Abteilungen gaben „superior" als bevorzugten Zugangsort an, 3 (17\%) bevorzugten „temporal" und 3 (17\%) „on axis“. Eine Übersicht über die bevorzugten Inzisionsgrößen befindet sich in Tab. 2. Es erfolgten 1609 Kataraktoperationen (3,04\%) unter systemischer Sedierung. Eine Übersicht über die Anästhesiemethoden befindet sich in Tab. 3 .

Im Jahr 2020 wurde an alle Abteilungen eine Antibiotikaprophylaxe durch intrakamerale Applikation am Operationsende verabreicht. Insgesamt wurde in 15 (0,029\%) Fällen von einer postoperativen Endophthalmitis berichtet.

Im Jahr 2020 wurden 370 Intraokularlinsen explantiert. Als Hauptgrund hierfür wurden IOL-Dislokation $(92 \%)$, Probleme mit Multifokallinsen (1\%), unerwünschte Refraktion (2\%) bzw. IOL-Trübungen (5\%) angegeben (Abb. 2).
Tab. 5 Verwendeter Linsentyp

\begin{tabular}{|l|l|l|l|l|l|l|l|l|l|}
\hline [Angaben in \%] & 2013 & 2014 & 2015 & 2016 & 2017 & 2018 & 2019 & 2020 \\
\hline Add on & 0,9 & 0,3 & 0,35 & 0,5 & 0,54 & 0,92 & 3 & 0,4 \\
\hline Asphärisch & 50 & 61,21 & 65,5 & 67,46 & 65,64 & 69,37 & - & 84,6 \\
\hline Blaufilter & 17,5 & 25,02 & 34,6 & 35,5 & 35,5 & 24,4 & 18 & 8,9 \\
\hline Multifokal & 0,3 & 0,4 & 0,8 & 0,2 & 0,6 & 1,2 & 1 & 0,5 \\
\hline Torisch & 1,1 & 1,42 & 2,8 & 4,02 & 7,8 & 3,8 & 5 & 5,5 \\
\hline
\end{tabular}

Die Dauer der postoperativen Medikation betrug im Durchschnitt in den meisten Fällen 2,9 Wochen (Abb. 3 und 4).

Die Aufteilung der verwendeten Implantate ist in Tab. 4 und 5 aufgelistet.

Danksagung Wir danken allen Abteilungen für ihre Mitarbeit.

Mitglieder der Kataraktkommission der ÖOG Dr. Egon Alzner; Prim. Univ. Prof. Dr. Michael Amon; Dr. Beatrix Neumaier-Ammerer; Dr. Brigitte Polonia; Prim. Univ. Prof. Dr. Oliver Findl; Univ. Prof. Dr. Claudette Abela-Formanek; Dr. Wolfgang Diem; Prim. Univ. Prof. Dr. Yosuf El-Shabrawi; PD Dr. Guenal Kahraman; Dr. Klaus Miller; Dr. Thomas Pfleger; Prim. Univ. Prof. Dr. Michael Radda; Ao. Univ. Prof. Dr. Wolfgang Radner; Ao. Univ. Prof. Dr. Georg Rainer; Dr. Markus Rossmann; Prim. Univ. Prof. Dr. Pia Vecsei-Marlovits.

Interessenkonflikt G. Kahraman gibt an, dass kein Interessenkonflikt besteht.

Hinweis des Verlags Der Verlag bleibt in Hinblick auf geografische Zuordnungen und Gebietsbezeichnungen in veröffentlichten Karten und Institutsadressen neutral. 